UNESUM-Ciencias: Revista Científica Multidisciplinaria

ANALISIS DE VARIABLES PARA MEDIR EFECTIVIDAD DE TUTORIAS EN INSTITUCION DE EDUCACION SUPERIOR

\title{
ANÁLISIS DE LAS VARIABLES PARA MEDIR LA EFECTIVIDAD DE TUTORIAS EN LA INSTITUCIÓN DE EDUCACIÓN SUPERIOR (IES)
} POLITÉCNICO INTERNACIONAL

\author{
AUTORES: Angelica Rocio Vargas Garay ${ }^{1}$ \\ Liliana García Barco² \\ Yesid Alexander Uchuvo González ${ }^{3}$
}

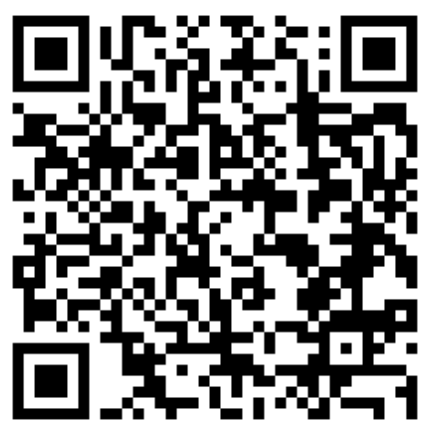

\section{DIRECCIÓN PARA CORRESPONDENCIA: angelica.vargasg@pi.edu.co}

Fecha de recepción: 12/01/2020

Fecha de aceptación: 21/03/2020

\section{RESUMEN}

Este proyecto se llevó a cabo en el marco del proceso de mejora continua de los diferentes programas de apoyo académico que ofrece la IES Politécnico Internacional. Uno de estos programas es el de tutorías en el cual los estudiantes reciben apoyo académico en las asignaturas que presentan mayores índices de mortalidad. El objetivo fundamental de este espacio radica en fortalecer las competencias académicas de los estudiantes para mejorar su rendimiento. Ello también contribuye a disminuir los índices de deserción. Sin embargo, la institución desconoce el nivel de importancia y la relación de cada una de las variables que influyen en la efectividad del programa. Por esta razón, el objetivo de la investigación consistió en analizar dichas variables para contribuir en la construcción a futuro de un indicador que permita dar cuenta de la efectividad. La Metodología del estudio correspondió a un análisis de tipo cuantitativo explicativo, con datos recolectados en una ventana de tiempo de un año. Para el estudio se tuvo en cuenta una población de 3.113 asistencias a tutorías y 1.129 estudiantes matriculados en el periodo 2018-1, 630 en el periodo 2018-2, 583 en el periodo 2018-3 y 443 en el periodo 2018-4. Se utilizó un muestreo a conveniencia del primer periodo. Esta información se organizó y codificó para su tratamiento estadístico en tres bases, que a su vez se convirtieron en categorías de análisis. Se observó que la influencia de la mayoría de los factores, alrededor de una tutoría, no se correlaciona de manera directa con la efectividad de esta. Es importante entender la complejidad del proceso tutorial ya

\footnotetext{
${ }^{1}$ IES Politécnico Internacional, Licenciada en Matemáticas, Maestría en Docencia e Investigación Universitaria. Grupo de Investigación: Educación y Sector Productivo.

${ }^{2}$ Universidad Colegio Mayor de Cundinamarca, Licenciada en Matemáticas, Maestría en Docencia e Investigación Universitaria.

${ }^{3}$ IES Politécnico Internacional, Licenciado en Humanidades con énfasis en español e inglés, Maestrante en Enseñanza de Lenguas Extranjeras. Grupo de Investigación: Educación y Sector Productivo
} 
Angelica Rocio Vargas Garay, Lilia Garay Barcia, Yesid Alexander Uchuvo González...

inmerso en la dinámica académica de la Institución para determinar un indicador que permita medir la efectividad de las tutorías.

PALABRAS CLAVE: efectividad, rendimiento académico, tutorías e indicador

\section{ANÁLISIS DE LAS VARIABLES PARA MEDIR LA EFECTIVIDAD DE LAS TUTORÍAS EN LA INSTITUCIÓN DE EDUCACIÓN SUPERIOR (IES) POLITÉCNICO INTERNACIONAL}

\section{ABSTRACT}

This research project was undertaken within the continual improvement framework of varied academic support programs offered by the Higher Education Institution called Politécnico Internacional. One of these academic support programs is named tutoring within which students receive academic support in the subjects concerning the highest rates of failure. The main aim of the tutorial program deals with strengthening the students' ${ }^{\prime}$ academic competences resulting in a better academic performance. This program also seeks to contribute to diminish the dropout rates. Nonetheless, the Institution do not know the importance and the relationship of the variables which may have an impact on the effectives of the tutoring program. Therefore, this research project aimed at analyzing those variables in order to contribute in the construction of an indicator that allows to account for the effectiveness of the program. The methodology of this study embraced a quantitative statistical explanatory analysis with gathered data in a year window time. The population of 3113 attendances and 1129 students for 2018-1, 630 students for 2018-2, 583 for 2018-3 and 443 for 2018-4 periods were considered to carry out this study. A convenient sample of the first period was utilized. The data were organized and coded in three data bases for the statistical treatment. It was observed that the influence of the majority of the factors surrounding a tutoring do not correlate the effectiveness of it directly. It is important to understand fully the complexity of the tutorial process immersed within the dynamic of the Institution to determine an indicator that allows to measure the effectiveness of the tutoring.

KEYWORDS: Effectiveness, academic performance, tutorials and indicator.

\section{INTRODUCCIÓN}

El estudio de la efectividad de los programas de apoyo académico en Educación Superior en Colombia cobra más relevancia en virtud de las pocas claridades que se tienen sobre las diferentes variables que potencialmente inciden en su ejecución, y, por lo tanto, en su mejora continua. Los factores que pueden estar asociados al programa de tutorías, uno de los programas de apoyo académico por antonomasia, están insertos en una amplia gama de variables cuya influencia no se ha dimensionado.

El Ministerio de Educación Nacional de Colombia (MEN,2009) establece que los programas de apoyo académico cuenten con un modelo de mejora continua en el que se monitoreen los avances, las necesidades y las posibles estrategias para robustecer la finalidad de cada programa. En este marco normativo cuasi autónomo, el estudio sobre la efectividad del programa de tutorías resulta 
UNESUM-Ciencias: Revista Científica Multidisciplinaria

ISSN 2602-8166

ANALISIS DE VARIABLES PARA MEDIR EFECTIVIDAD DE TUTORIAS EN INSTITUCION DE EDUCACION SUPERIOR

fundamental no solo para la mejora continua institucional, sino también para el afinamiento del objetivo del programa.

A lo largo del funcionamiento del Programa Institucional de Tutorías en la IES Politécnico Internacional se ha entendido la efectividad de este como la comparación de los porcentajes de aprobación de una asignatura con relación a la asistencia a las tutorías, teniendo como referente un porcentaje establecido por la institución. Sin embargo, la institución desconoce el nivel de importancia y la relación de cada una de las variables que influyen en la efectividad del programa. Al hacer un análisis de las variables que intervienen, y las relaciones entre ellas, se podría construir un indicador que permitiera medir la efectividad de las tutorías.

En este contexto, la pregunta que direcciona el presente estudio es ¿Cómo determinar la efectividad de las tutorías en la IES Politécnico Internacional?

\section{DESARROLLO}

\section{MARCO CONCEPTUAL}

\section{Los tipos de tutoría y una aproximación a la efectividad.}

En el contexto colombiano, resulta fundamental resaltar los estudios enmarcados en la educación superior con relación a las tutorías entendidas como apoyo y/o estrategia académica. Según Cardozo (2011) la mayoría de los estudios asociados a las tutorías universitarias gravitan en torno al acompañamiento del profesor al estudiante, particularmente enfatizado en el marco de la educación a distancia (p. 310). En esta misma línea, es evidente que muy poco se ha ahondado en el tema de la efectividad; dado que la tutoría obedece a la necesidad de mejorar el rendimiento académico de los estudiantes, constituye, para el presente estudio, fundamental identificar y analizar las diferentes variables involucradas en el proceso de una tutoría, y a partir de allí, dar luces para creación de indicador que permita a la Institución medir la efectividad de dicho programa.

La tutoría se ha definido desde varias perspectivas, por un lado, se encuentran las tutorías académicas, las cuales adquieren relevancia en la formación disciplinaria, entendiendo estas como "una actividad de carácter formativo que incide en el desarrollo integral de los estudiantes universitarios en su dimensión intelectual, académica, profesional y personal. (Ferrer, 2003: 67). Lobato y Guerra (2014, p.18) afirman que la tutoría académica es fundamental en el contexto universitario porque se visualiza como "la intervención educativa personalizada de acompañamiento, asesoramiento y apoyo en la adquisición y maduración de las competencias y en la configuración del proyecto personal y profesional del estudiante".

En este mismo sentido, Cano (2009) sostiene que la tutoría debiera ser el elemento básico de calidad educativa, expresada en sus diversas formas (académica, personal y/o profesional) y en sus diferentes manifestaciones (individual, 
Angelica Rocio Vargas Garay, Lilia Garay Barcia, Yesid Alexander Uchuvo González...

grupal y entre pares), pues todas ellas son complementarias entre sí. De acuerdo a lo anteriormente mencionado se define la tutoría académica como una actividad de carácter formativo que incide en el desarrollo integral de los estudiantes universitarios en su dimensión intelectual, académica, profesional y personal (Ferrer, 2003).

Retomando al mismo autor, se proponen dos fases metodológicas para las tutorías académicas:

1. Fase: Reflexión sobre la integración teórico-práctica. Identificación y evaluación de los aprendizajes relevantes en torno a su quehacer profesional.

2. Fase: Se activan los conocimientos previos de los estudiantes y se contextualizan en la práctica, para identificar, sistematizar, deconstruir y evaluar los procesos de aprendizaje alcanzados, y desarrollar acciones de mejora.

La tutoría tiene una influencia en dos grandes frentes que no son necesariamente dependientes, a saber: Rendimiento académico y permanencia estudiantil. "La tutoría como intervención formativa destinada al seguimiento académico de los estudiantes es una estrategia pedagógica ...como una manera de brindar respuesta a la problemática de deserción" (Cardozo, 2011, p. 322). Lo anterior también se puede ver reflejado en el análisis suministrado por el Sistema para la Prevención de Deserción en Instituciones de Educación Superior (SPADIES) del Ministerio de Educación Nacional de Colombia (MEN) en relación con los factores asociados al fenómeno de la deserción. Entre los más importantes se encuentran:

“Tinto midió, en sus estudios, la integración académica mediante las calificaciones del estudiante y la integración según la frecuencia de interacciones de este con sus pares y con los docentes. Determinó que, cuando estas dos dimensiones se evalúan positivamente, se refuerzan la persistencia del estudiante y su grado de compromiso con la institución” (MEN, 2015. p, 99)

Por otro lado, encontramos la tutoría entre iguales, la cual se define como un método de aprendizaje cooperativo que se basa en la creación de parejas de alumnado con una relación asimétrica y que comparten un mismo objetivo (Duran, \& Vidal, 2004). La tutoría entre iguales es una de las prácticas instructivas más efectivas para la educación de calidad (Ainscow, 1991; Echeita, 2006; Stainback \& Stainback, 2001). Las prácticas de tutoría entre iguales permiten variedad de situaciones dependiendo de criterios relativos al contenido, formato, edad, continuidad del rol, tiempo o características de los participantes (Topping, 1996). Dentro de una tutoría entre iguales, replicando al autor, los participantes pueden ejercer distintos roles (tutor o tutorado), y se puede dar de dos maneras:

Fija: El papel que toma cada participante se mantiene estable durante toda la tutoría.

Recíproca: Se realiza un intercambio de roles durante algunas sesiones de la tutoría.

En ambos casos tanto el tutor como el tutorado se benefician del espacio, por un lado, el tutor realiza un proceso de aprendizaje al enseñar, y por el otro lado el tutorado recibe los aprendizajes personalizados que le brinda el tutor. La interacción uno a uno que ofrece la tutoría entre iguales permite que los miembros de la pareja se ofrezcan ayudas pedagógicas andamiadas, donde el tutor -reconociendo la zona de desarrollo del tutorado le ofrece ayudas ajustadas

182 UNESUM-Ciencias. Publicación cuatrimestral. Vol. 4, Año 2020, No. 1 (Enero - Abril) 
UNESUM-Ciencias: Revista Científica Multidisciplinaria

ISSN 2602-8166

ANALISIS DE VARIABLES PARA MEDIR EFECTIVIDAD DE TUTORIAS EN INSTITUCION DE EDUCACION SUPERIOR

(insinuar, apuntar, ajustar, extraer información, resumir) (Graesser, D’Melo, \& Cade, 2011; Graesser \& Person, 1994).

Aparece entonces la explicación más allá de la simple trasmisión de conocimiento.

Dada la importancia del espacio de tutoría en las instituciones de educación superior, surge la necesidad de no solo esclarecer las variables que intervienen sino también analizarlas de tal manera que el análisis pueda contribuir a la construcción de un indicador que permita medir el grado de efectividad de esta, en dichos términos es indispensable establecer la diferencia entre efectividad, eficiencia y eficacia.

Según Mejía (2015), los indicadores de efectividad y eficacia, en cualquier área de una organización, permiten definir un resultado esperado, el cual se puede expresar como: meta, cantidad, variación, porcentaje, etc. De igual manera, se contempla un costo estimado y un tiempo especificado, y de esta manera al combinar dichos valores se puede medir objetivamente el grado de efectividad y eficiencia de un área de dicha organización. Para el propósito de este estudio se tomó a la efectividad como el alcance del objetivo trazado por el programa de tutorías.

\section{Tutorías en la IES Politécnico Internacional}

El Politécnico Internacional fundamenta la concepción de la tutoría a partir del horizonte institucional demarcado por la misión, la visión y los principios que regulan su funcionamiento, tal como lo sugiere el MEN (2015) en la guía para la implementación del Modelo de Gestión de Permanencia y Graduación Estudiantil en Instituciones de Educación Superior. Así pues, una tutoría es entendida como un proceso de acompañamiento y apoyo integral en las dimensiones personales, académicas y profesionales que abarca: orientación (vocacional), motivación y persistencia (para la continuidad de los planes de estudio), apoyo académico focalizado y particularizado para la superación de dificultades de aprendizaje (que deberá garantizar el consecuente logro de la competencia), así como un espacio de práctica para el dominio de una habilidad-desempeño particular.

El Politécnico Internacional pone a disposición de la comunidad académica 4 tipos de tutorías, a saber: Académica, Personal, de Reentrenamiento y Talleres de Práctica.

\section{MARCO METODOLÓGICO}

\section{Enfoque y tipo de investigación:}

El presente estudio es de tipo cuantitativo explicativo (Sampieri at al., 2006), pasando por dos fases: organización de la información y análisis descriptivo correlacional, ya que para la consecución del objetivo de la investigación (analizar las variables y la relación entre ellas) se deben organizar, identificar y clasificar los factores que inciden en la efectividad de las tutorías para luego realizar el análisis descriptivo y establecer la correlación entre las variables. 
Angelica Rocio Vargas Garay, Lilia Garay Barcia, Yesid Alexander Uchuvo González...

El proceder de la primera fase de la investigación consistió en tomar la información recolectada y organizarla de modo que las variables fueran congruentes con el objetivo trazado. Este proceso continuó con la depuración de datos incompletos y la consecuente exclusión de tres tipos de tutorías: Talleres Prácticos, Personales y de Reentrenamiento, a razón de que la tutoría de tipo Académica, la cual es analizada, se presenta como un fenómeno mucho más complejo en su funcionamiento. Los múltiples factores que hacen parte de ésta permiten su problematización y, por lo tanto, ponen de pleno un interrogante a ser resuelto. Acto seguido, se realizó una selección de las variables relevantes para el objeto del estudio, tales como el número de tutorías, frecuencia de asistencia y aspectos sociodemográficos de los actantes, entre otros. Es así como se procedió a establecer las siguientes categorías de análisis:

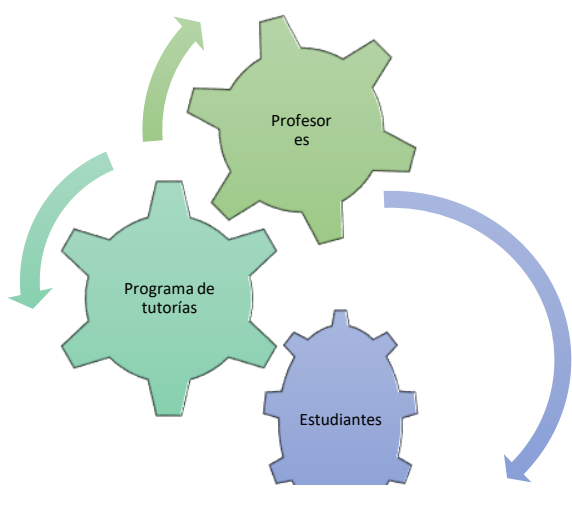

Figura 1Categorías de análisis

Fuente: elaboración propia

Para fines del análisis descriptivo y correlacional de las variables identificadas, se hizo uso del programa estadístico SPSS. En tanto al análisis descriptivo se realizaron tablas y gráficos que permitieron sintetizar la información priorizando las variables que mostraron un comportamiento relevante para el objetivo de la información. Posteriormente, se utilizó el coeficiente de correlación de Pearson y la prueba de Chi-cuadro para determinar la relación de dependencia entre las diferentes variables. Finalmente, se dio pasó al análisis de estos resultados para darle alcance al objetivo del estudio.

\section{Contexto, población y muestra}

El estudio se llevó a cabo en la IES Politécnico Internacional (PI) de la sede Principal (Av. Chile) Bogotá, Colombia. El PI es de carácter técnico-tecnológico y su principal población objetivo está circunscrita a los estratos menos favorecidos. El PI ofrece 4 tipos de tutoría, pero para el presente estudio solo se hizo uso de la tutoría Académica previamente referenciada. La población corresponde a 3.113 asistencias a tutorías Académicas y 1.129 estudiantes matriculados en el periodo 2018-1, 630 en el periodo 2018-2, 583 en el periodo 2018-3 y 443 en el periodo 2018-4. Se utilizó un muestreo a conveniencia correspondiente al periodo 2018-1, en el cual se presentaron 1.294 asistencias y 1.129 estudiantes. Se tomó esta muestra en virtud de la mayor representatividad de la información y la facilidad para su manejo debido al gran número de 

EDUCACION SUPERIOR

datos a ser analizados. De igual manera, se consideró que todos los elementos de la población podrían haber sido parte del estudio, ya que todos cumplían con las características necesarias para su análisis.

\section{Instrumentos de recolección de información}

En el proceso investigativo se utilizaron tres fuentes de información suministradas por tres áreas de la Institución. En la primera base se consolidó la información generada por los tutores y estudiantes asistentes durante los espacios de tutorías. Esta base de asistencias son el resultante de un cuestionario en el que se pregunta por los datos básicos de los participantes en el proceso tutorial. La segunda fuente de información corresponde a una base datos, la cual fue suministrada por el área de Registro y Control, que contiene la información sociodemográfica y académica de los estudiantes. La tercera, proporcionada por el área de Gestión Humana, se relaciona con la información de los docentes tutores en tanto formación, salarios, intensidad horaria y algunos aspectos sociodemográficos.

\section{RESULTADOS}

La muestra para el periodo 2018-1 es de 1.129 estudiantes de los cuales el 65,7\% (731 estudiantes) no asistieron a ninguna tutoría, mientras que el 35,3\% (398 estudiantes) restante asistió, para un total de 728 asistencias. Para el presente análisis se tuvo en cuenta solo a los estudiantes asistentes a una sola materia de tutoría cuya frecuencia osciló entre 1 a 7 asistencias.

De los estudiantes asistentes tenemos que: $55,7 \%$ son mujeres y $44,2 \%$ hombres. El $88 \%$ pertenece a los estratos 1, 2 y 3. EL 65\% no labora en comparación con el 35\% que labora. El $58 \%$ pertenece al ciclo 1 del programa académico y el 54\% pertenecen a la jornada diurna. Además, las edades donde se concentra la mayor cantidad de los estudiantes corresponden a edades entre los 16 y 22 años, como se muestra en la Figura 2. 
Angelica Rocio Vargas Garay, Lilia Garay Barcia, Yesid Alexander Uchuvo González...

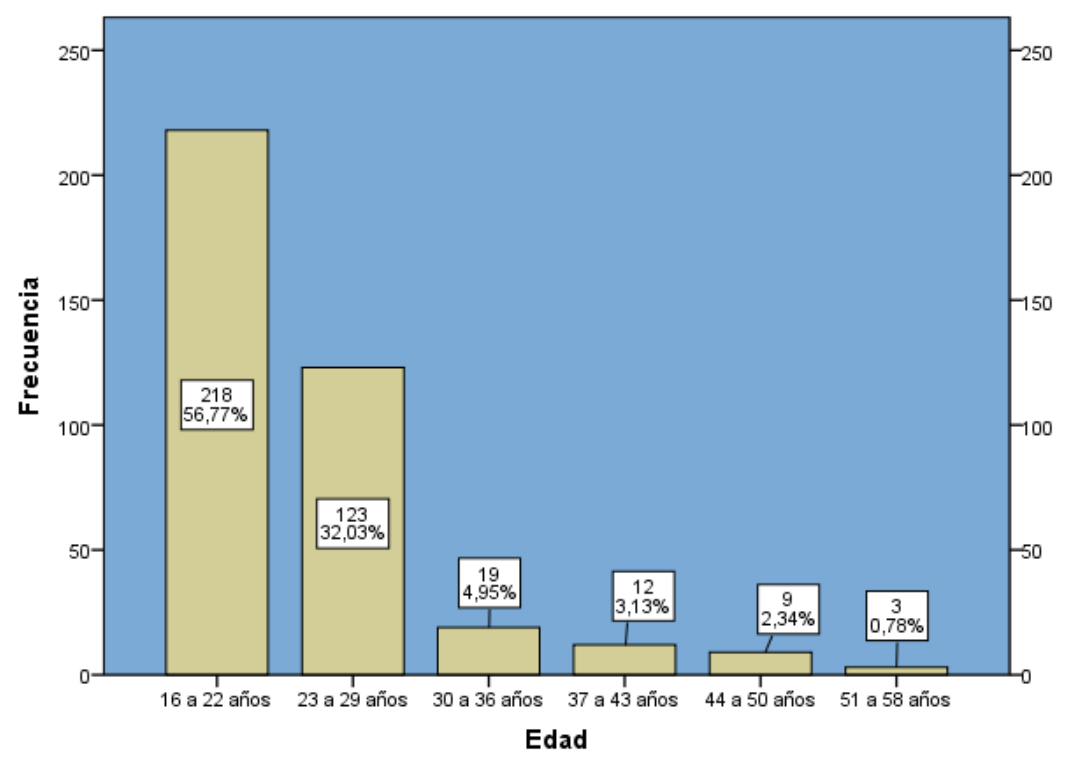

Figura 2Edad de los estudiantes que asisten periodo 2018-1

Fuente: elaboración propia

Se puede evidenciar que la mayoría de los estudiantes que asisten corresponden a las facultades de Hospitalidad y Emprendimiento ( ver Figura 3)

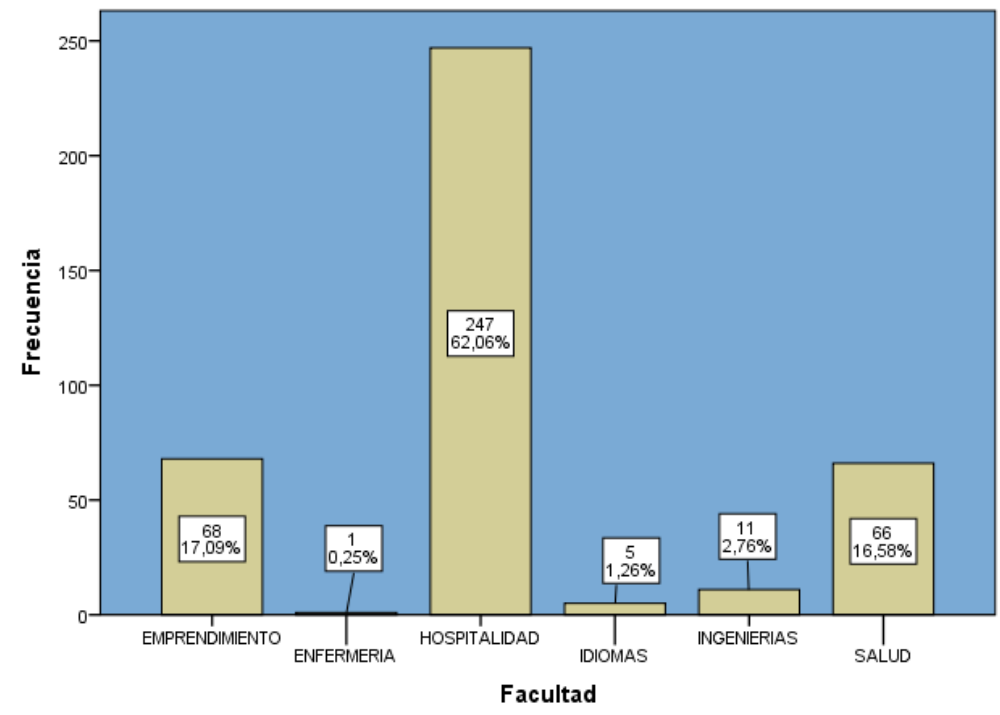

2018-1

Figura 3Estudiantes asistentes por facultad periodo

elaboración propia

Se evidencia, como muestra la Figura 4, que el 42,2\% de asistentes fueron reportados como producto de una remisión y de estos aprobaron $88.09 \%$ (148 estudiantes). Mientras que se observa que de los estudiantes asistentes voluntariamente $57.79 \%$, aprobaron $94.78 \%$ (218 estudiantes) 


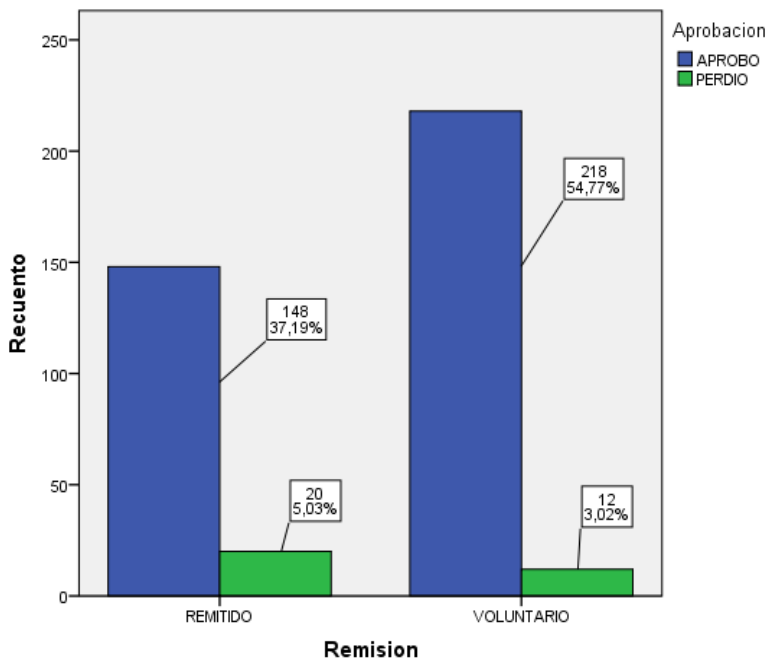

Figura 4 Tasa de aprobación de los estudiantes remitidos vs voluntarios

Fuente: elaboración propia

Según la Figura 5, se evidenció que a medida que aumentan el número de asistencias a tutoría por estudiante disminuye el porcentaje de pérdida de la asignatura correspondiente a la tutoría.

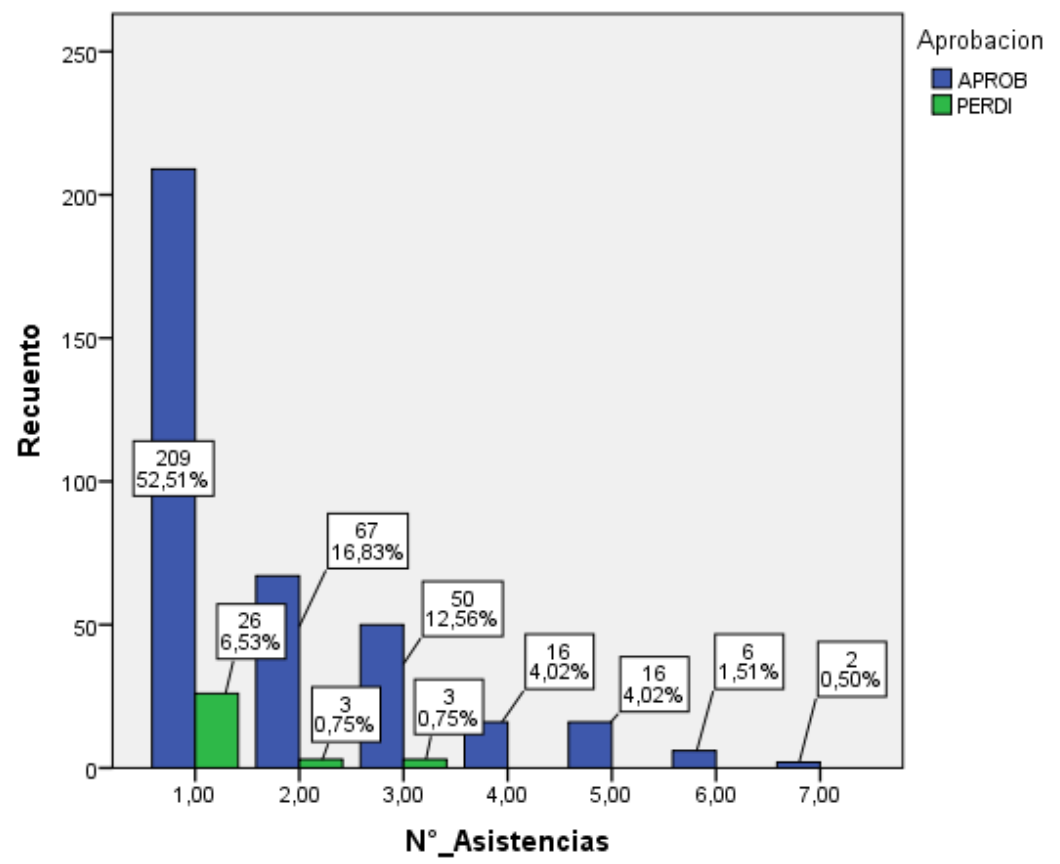

Figura 5 Aprobación vs asistencias periodo 2018 -

1 
Angelica Rocio Vargas Garay, Lilia Garay Barcia, Yesid Alexander Uchuvo González...

En cuanto a los docentes de las diferentes tutorías, los resultados para este periodo muestran que la mayoría de los estudiantes que asisten a su respectiva tutoría aprueba en mayor proporción como se muestra en la tabla 1. En total el $91.96 \%$ aprueban la asignatura a su correspondiente tutoría.

Tabla 1. Aprobación vs Docentes Tutores periodo 2018-1

\begin{tabular}{|c|c|c|c|c|}
\hline & & \multicolumn{2}{|c|}{ Aprobación } & \multirow[t]{2}{*}{ Total } \\
\hline & & Aprobó & Perdió & \\
\hline \multirow{15}{*}{ Docente Tutor } & Angela Jaimes & 19 & 3 & 22 \\
\hline & Angelica Vargas & 4 & 0 & 4 \\
\hline & Hernando Almanza & 1 & 0 & 1 \\
\hline & Jim Soto & 3 & 3 & 6 \\
\hline & Jimmy Aldana & 10 & 3 & 13 \\
\hline & Jorge Rincon & 69 & 1 & 70 \\
\hline & Laura Trujillo & 16 & 0 & 16 \\
\hline & Lotus Salcedo & 10 & 0 & 10 \\
\hline & Manuel Duarte & 35 & 2 & 37 \\
\hline & Marcela Benitez & 1 & 0 & 1 \\
\hline & Marcela Ramirez & 65 & 8 & 73 \\
\hline & Meribe Soto & 57 & 2 & 59 \\
\hline & Nelson David Arango & 4 & 0 & 4 \\
\hline & Rafael Cedeño & 34 & 7 & 41 \\
\hline & Sergio Morales & 38 & 3 & 41 \\
\hline Total & & & & \\
\hline
\end{tabular}

Fuente: elaboración propia

Otro de los resultados relevantes se relaciona con las notas de los asistentes a tutorías. Como se evidencia en la Tabla 1, los porcentajes de aprobación más altos se manifiestan en el caso de los estudiantes asistentes a tutorías con valor de $92 \%$ en contraste con los no asistentes cuyo porcentaje de aprobación es de 75,5\%.

Tabla 2 Nota asignatura vs asistencia a la tutoría

\begin{tabular}{|c|c|c|c|c|c|}
\hline & & & \multicolumn{2}{|c|}{ Asiste } & \multirow[t]{2}{*}{ Total } \\
\hline & & & NO & sí & \\
\hline \multirow{5}{*}{ Nota } & 0,0 a 0,9 & $\begin{array}{l}\text { Recuento } \\
\text { Porcentaje }\end{array}$ & $\begin{array}{c}45 \\
6,2 \%\end{array}$ & $\begin{array}{c}12 \\
3,0 \%\end{array}$ & $\begin{array}{c}57 \\
5,0 \%\end{array}$ \\
\hline & 1,0 a 1,9 & $\begin{array}{l}\text { Recuento } \\
\text { Porcentaje }\end{array}$ & $\begin{array}{c}77 \\
10,5 \%\end{array}$ & $\begin{array}{c}8 \\
2,0 \%\end{array}$ & $\begin{array}{c}85 \\
7,5 \%\end{array}$ \\
\hline & 2,0 a 2,9 & $\begin{array}{l}\text { Recuento } \\
\text { Porcentaje }\end{array}$ & $\begin{array}{c}56 \\
7,7 \%\end{array}$ & $\begin{array}{c}12 \\
3,0 \%\end{array}$ & $\begin{array}{c}68 \\
6,0 \%\end{array}$ \\
\hline & 3,0 a 3,9 & $\begin{array}{l}\text { Recuento } \\
\text { Porcentaje }\end{array}$ & $\begin{array}{c}236 \\
32,3 \%\end{array}$ & $\begin{array}{c}154 \\
38,7 \%\end{array}$ & $\begin{array}{c}390 \\
34,5 \%\end{array}$ \\
\hline & 4,0 a 5,0 & $\begin{array}{l}\text { Recuento } \\
\text { Porcentaje }\end{array}$ & $\begin{array}{c}317 \\
43,4 \%\end{array}$ & $\begin{array}{c}212 \\
53,3 \%\end{array}$ & $\begin{array}{c}529 \\
46,9 \%\end{array}$ \\
\hline \multicolumn{2}{|c|}{ Total } & Recuento & 731 & 398 & 1129 \\
\hline
\end{tabular}

188 UNESUM-Ciencias. Publicación cuatrimestral. Vol. 4, Año 2020, No. 1 (Enero - Abril) 


\begin{tabular}{|l|l|l|l|l|}
\hline & Porcentaje & $64,7 \%$ & $35,3 \%$ & $100,0 \%$ \\
\hline
\end{tabular}

Fuente: elaboración propia

Dentro del análisis correlacional, se realizaron diversos cruces entre variables que arrojaron una independencia entre los diferentes factores. Por ejemplo, al correlacionar la frecuencia con la que un estudiante va a la tutoría versus la aprobación, los porcentajes se puede observar en la Tabla 3; además los resultados del valor p de la prueba es de 0.228 (ver Tabla 4) cuya hipótesis nula es que la aprobación de la materia objeto de la tutoría es independiente a la frecuencia con la que un estudiante asiste a la tutoría; pero como el valor p es mayor a 0,05, esto empleando la prueba de Chi-cuadrado de Pearson no se rechaza la hipótesis nula por lo tanto la aprobación es independiente de la frecuencia con que asisten los estudiantes a la tutoría.

Tabla 3 Frecuencia asistencia a las tutorías vs Aprobación

\begin{tabular}{|c|c|c|c|c|c|}
\hline & & & Aprc & & \\
\hline & & & Aprobación & Perdió & Total \\
\hline & 1,00 & Recuento & 209 & 26 & 235 \\
\hline & & $\%$ del total & $52,5 \%$ & $6,5 \%$ & $59,0 \%$ \\
\hline & 2,00 & Recuento & 67 & 3 & 70 \\
\hline & & $\%$ del total & $16,8 \%$ & $0,8 \%$ & $17,6 \%$ \\
\hline & 3,00 & Recuento & 50 & 3 & 53 \\
\hline & & $\%$ del total & $12,6 \%$ & $0,8 \%$ & $13,3 \%$ \\
\hline Frecuencias & 4,00 & Recuento & 16 & 0 & 16 \\
\hline asistencias & & $\%$ del total & $4,0 \%$ & $0,0 \%$ & $4,0 \%$ \\
\hline & 5,00 & Recuento & 16 & 0 & 16 \\
\hline & & $\%$ del total & $4,0 \%$ & $0,0 \%$ & $4,0 \%$ \\
\hline & 6,00 & Recuento & 6 & 0 & 6 \\
\hline & & $\%$ del total & $1,5 \%$ & $0,0 \%$ & $1,5 \%$ \\
\hline & 7,00 & Recuento & 2 & 0 & 2 \\
\hline & & $\%$ del total & $0,5 \%$ & $0,0 \%$ & $0,5 \%$ \\
\hline & & Recuento & 366 & 32 & 398 \\
\hline 100 & & $\%$ del total & $92,0 \%$ & $8,0 \%$ & $100,0 \%$ \\
\hline
\end{tabular}

Fuente: elaboración propia

Tabla 4 Pruebas de Chi-cuadrado frecuencia asistencias vs aprobación

\begin{tabular}{|l|c|c|c|}
\hline & Valor & df & $\begin{array}{c}\text { Significación } \\
\text { asintótica } \\
\text { (bilateral) }\end{array}$ \\
\hline Chi-cuadrado de Pearson & $8,144^{\mathrm{a}}$ & 6 &, 228 \\
\hline Razón de verosimilitud & 11,367 & 6 &, 078 \\
\hline N de casos válidos & 398 & & \\
\hline
\end{tabular}

Fuente: elaboración propia 
Angelica Rocio Vargas Garay, Lilia Garay Barcia, Yesid Alexander Uchuvo González...

Otra de las variables que se compararon y resultaron independientes son las notas y la frecuencia con la que un estudiante va a la tutoría. Para determinar independencia de estas variables se utilizó la prueba de Chi-cuadrado de Pearson cuyo valor p de la prueba fue de 0,649, (ver Tabla 5). Así, el resultado es no rechazar la hipótesis nula, que la aprobación de la materia objeto de la tutoría es independiente a la frecuencia con la que un estudiante asiste.

Tabla 5 Pruebas de Chi-cuadrado frecuencia asistencia vs notas

\begin{tabular}{|l|c|c|c|}
\hline & Valor & df & $\begin{array}{c}\text { Significación } \\
\text { asintótica } \\
\text { (bilateral) }\end{array}$ \\
\hline Chi-cuadrado de Pearson & $20,821^{\text {a }}$ & 24 &, 649 \\
\hline Razón de verosimilitud & 28,621 & 24 &, 235 \\
\hline Asociación lineal por lineal & 11,249 & 1 &, 001 \\
\hline N de casos válidos & 398 & & \\
\hline
\end{tabular}

Fuente: elaboración propia

En esta misma línea, se encontró que otras de las variables independientes son la nota en comparación con la edad y la remisión a tutorías con relación a la nota. No obstante, fue posible hallar que, como muestra la Tabla 6, de acuerdo con la prueba de chi-cuadrado de Pearson con un valor de ji cuadrado de 47,59 y 4 grados de libertad, cuyo valor p es de 0,00 menor a 0,05 se rechaza la hipótesis nula, es decir, la nota depende de la asistencia a tutorías y las mayores notas corresponden a los que sí asistieron a tutorías.

Tabla 6 Pruebas de Chi-cuadrado asistencia vs nota

\begin{tabular}{|l|c|c|c|}
\hline & Valor & df & $\begin{array}{c}\text { Significación } \\
\text { asintótica } \\
\text { (bilateral) }\end{array}$ \\
\hline Chi-cuadrado de Pearson & $47,591^{\text {a }}$ & 4 &, 000 \\
\hline Razón de verosimilitud & 54,698 & 4 &, 000 \\
\hline N de casos válidos & 1129 & & \\
\hline
\end{tabular}

Fuente: elaboración propia

\section{CONCLUSIONES}

El programa de tutorías académicas en la IES Politécnico Internacional como fenómeno complejo en el cual intervienen diversas variables resulta ser efectivo en la medida en que contribuye en el proceso académico evidenciado en la aprobación de la asignatura que impacta la tutoría per se. Ahora bien, aunque la mayoría de las variables analizadas son independientes, se puede evidenciar una influencia positiva de la variable asistencia que podría interpretarse en términos de la calidad de una tutoría y no en términos de la frecuencia de asistencias. 
UNESUM-Ciencias: Revista Científica Multidisciplinaria

ANALISIS DE VARIABLES PARA MEDIR EFECTIVIDAD DE TUTORIAS EN INSTITUCION DE EDUCACION SUPERIOR

A través de este estudio se puedo hallar que las variables que intervienen el todo el proceso tutorial son mucho más complejas de comprender y, por lo tanto, de ser analizadas. Esto podría implicar para posteriores estudios la utilización de la categorización emergente en esta investigación. Se advierte que tanto la recolección como la organización de la información resultan ser factores determinantes para dilucidar la efectividad de una tutoría, además de contribuir a la construcción de un indicador que permita la identificación y aplicación de estrategias en vía de la mejora continua del programa de tutorías.

Para posteriores estudios podría resultar fundamental ahondar en la comprensión y subsecuente análisis de variables tan complejas como la facultad de la que proviene el estudiante y las remisiones como factores que permitan dilucidar desde otros ángulos la efectividad de las tutorías.

\section{REFERÉNCIAS BIBLIOGRÁFICAS}

Andreucci, P. \& Curiche, A. (2017). Tutorías académicas: Desafíos de un programa piloto entre pares en una universidad no selectiva. Revista Latinoamericana de Ciencias Sociales, Niñez y Juventud, 15 (1), 357-371

Álvarez, P. \& Pérez, C. Tutoría universitaria como respaldo al aprendizaje y construcción del proyecto formativo del alumnado. Mèxico Julio-Diciembre 2016. Revista Mexicana de Orientación Educativa is the property of Centro de Investigacion y Formacion para la Docencia y Orientacion Educativa, S.C. (CENIF, S.C.)

Cano, R. (2009). Tutoría universitaria y aprendizaje por competencias. ¿Cómo lograrlo?. Revista Electrónica Interuniversitaria de Formación del Profesorado, 12 (1), 181-204.

Cardozo, C. \& Ortiz M.Tutoría entre pares como una estrategia pedagógica universitaria. ISSN 0123-1294 Educ.Educ. Vol. 14, No. 2 | Mayo-agosto de 2011 | pp. 309-325

Dolores, G., Lima, S. \& Lima, R. (2017). Efectividad de las tutorías para mejorar el rendimiento académico de los estudiantes de Enfermería de la Universidad de Sevilla. JOURNAL OF NEW APPROACHES IN EDUCATIONAL RESEARCH Vol. 6. No. 2. pp. 93-103 ISSN: 2254-7339.

Sampieri, R. H., Collado, C. F., \& Lucio, P. B. (2006). Metodología de la investigación. New York, NY: McGrawHill Companies.

Ministerio de Educación Nacional, MEN (2009). Deserción estudiantil en la educación superior colombiana. Metodología de seguimiento, diagnóstico y elementos para su prevención. República de Colombia. Disponible en www.mineducacion.gov.co

Ministerio de Educación Nacional, MEN (2015). Estrategias para la permanencia en educación superior: experiencias significativas. República de Colombia. Disponible en www.mineducacion.gov.co

Ministerio de Educación Nacional, MEN (2016). Documento de lineamientos de política pública. Sistema Nacional de Educación Terciaria (SNET): Camino para la inclusión, la equidad y el reconocimiento. República de Colombia. Disponible en www.mineducacion.gov.co

Rosado, M (2006). Metodología de investigación y evaluación. Editorial Trillas.

Stake, R (2007). Investigación con estudio de casos. Madrid: Edcs. Morata. Cuarta edición. Morata. Madrid, España.

Viale, H. (2014). Una aproximación teórica a la deserción estudiantil universitaria. Revista Digital de Investigación en Docencia Universitaria. Año 8. Número 1.

Yin, R. (2009). Case study research. Design and methods. Sage publications. Thousand oaks. 4th 